\title{
ANÁLISE DA MARGEM CIRÚRGICA PROXIMAL DO ESTÔMAGO EM PACIENTES SUBMETIDOS À GASTRECTOMIA SUBTOTAL POR ADENOCARCINOMA
}

\section{Proximal gastric margin analysis following subtotal gastrectomy for adenocarcinoma}

\author{
Alexandre Coutinho Teixeira de FREITAS, Josiane Mourão DIAS, Luciano OKAWA, Júlio Cezar Uili COELHO
}

ABCDDV/604

Freitas ACT, Dias JM, Okawa L, Coelho JCU. Análise da margem cirúrgica proximal do estômago em pacientes submetidos à gastrectomia subtotal por adenocarcinoma. ABCD Arq Bras Cir Dig 2008;21(3):106-9

RESUMO - Racional - A gastrectomia subtotal atualmente é considerada padrão ouro no tratamento da neoplasia gástrica do terço médio e distal. No entanto, foi demonstrado que a ocorrência de neoplasia residual na margem cirúrgica proximal está associada à redução da sobrevida. Objetivos analisar a margem cirúrgica proximal no exame anátomo-patológico de pacientes submetidos à gastrectomia subtotal por adenocarcinoma gástrico e identificar os fatores relacionados com o acometimento neoplásico dessa margem. Métodos - No período entre janeiro de 1998 e dezembro de 2007 foram revisados os prontuários dos pacientes submetidos à gastrectomia subtotal devido a adenocarcinoma gástrico do terço médio e distal. Os pacientes foram analisados quanto à idade, sexo, classificação de Lauren, classificação de Borrmann, maior diâmetro da lesão, localização da lesão no estômago, clínico e presença de invasão angiolinfática. Foi realizada análise univariada desses dados em relação ao acometimento da margem proximal do estômago no exame anátomo-patológico. Resultados - Foram analisados 104 casos: 34 do sexo feminino e 70 do sexo masculino com idade média de $57 \pm 13$ anos. Doze pacientes (12,3\%) apresentaram acometimento da margem proximal. A análise univariada entre os fatores analisados e o acometimento neoplásico da margem proximal demonstrou associação somente em relação à classificação de Borrmann. Conclusão - A classificação macroscópica de Borrmann, especialmente nos estágios III e IV, está relacionada à presença de acometimento da margem proximal nos casos de adenocarcinoma gástrico dos terços médio e distal submetidos a tratamento com gastrectomia subtotal.

DESCRITORES - Neoplasias gástricas. Adenocarcinoma. Gastrectomia. Neoplasia residual.

\section{INTRODUÇÃO}

O câncer gástrico é a quarta neoplasia mais comum no mundo ${ }^{6}$.Aproximadamente 600.000 casos são diagnosticados anualmente e cerca de dois terços dos pacientes morrem apesar do tratamento utilizado ${ }^{6}$. O adenocarcinoma corresponde a $95 \%$ de todas as neoplasias malignas do estômago $^{10}$.

Apesar da tentativa de desenvolvimento de diversas terapias para o câncer gástrico, a cirurgia ainda é o único procedimento capaz de curar esse tipo de doença. Atualmente, reconhece-se a gastrectomia total como padrão ouro no tratamento da neoplasia gástrica localizada no terço proximal do estômago ${ }^{3,10,12}$. A gastrectomia subtotal é utilizada para tratamento das lesões localizadas no terço médio e distal. Nesse procedimento deve-se respeitar margem de segurança proximal de cinco a seis centímetros nos casos de neoplasia avançada e de dois nos de neoplasia precoce ${ }^{1,2,10,12,14}$. Normalmente existe preocupação maior em relação à margem distal. Devido ao número significa-

Trabalho realizado no Serviço de Cirurgia do Aparelho Digestivo do Departamento de Cirurgia da Universidade Federal do Paraná, Curitiba, PR, Brasil.

Correspondência: Alexandre Coutinho Teixeira de Freitas, e-mail: alexandrefreitas@ufpr.br tivo de tumores com localização mais distal no estômago, a obtenção de segmento duodenal livre de neoplasia é tarefa tecnicamente mais frequente e difícil. No entanto, também existe possibilidade de acometimento da margem cirúrgica proximal. Esse acometimento, mesmo que microscópico, cursa com redução na sobrevida ${ }^{9,13,4}$. Cho et al. ${ }^{4}$ demonstraram sobrevida média de 34 meses em 49 pacientes com margem comprometida e sobrevida de 69 meses em 2691 pacientes com margens livres.

O desenvolvimento de mecanismos que tornem possível evitar ou diagnosticar a possibilidade de neoplasia residual na margem proximal é de grande importância. Algumas recomendações são encontradas na literatura médica, porém a identificação de fatores de risco que possam prever essa possibilidade seria de grande utilidade.

Esse estudo tem como objetivo analisar a margem cirúrgica proximal no exame anátomo-patológico de pacientes submetidos à gastrectomia subtotal por adenocarcinoma gástrico e identificar os fatores relacionados com o acometimento neoplásico dessa margem.

\section{MÉTODOS}

Foram revisados os prontuários e os laudos do exame de anatomia patológica dos pacientes submetidos à gas- 
trectomia subtotal aberta por adenocarcinoma gástrico no Hospital de Clínicas da Universidade Federal do Paraná no período compreendido entre janeiro de 1998 e dezembro de 2007. Os pacientes foram analisados quanto à idade, sexo, classificação de Lauren e de Borrmann, maior diâmetro da lesão, localização da lesão no estômago, clínico e presença de invasão angiolinfática. Foi realizada análise univariada desses dados em relação ao acometimento da margem proximal do estômago. Quanto ao clínico essa análise foi realizada em relação ao estádio propriamente dito e também em relação às suas subdivisões em $\mathrm{T}$ (profundidade da lesão), $\mathrm{N}$ (acometimento de linfonodos) e M (presença de metástases). Em relação ao diâmetro da neoplasia a análise do acometimento da margem proximal foi realizada comparando-se os portadores de neoplasia com diâmetro menor ou igual a dois centímetros com os com diâmetro maior do que dois centímetros. Também foi analisado o acometimento da margem proximal com relação às neoplasias com diâmetro maior e às com menor do que quatro centímetros. Também foi realizada análise estatística da relação entre a classificação de Lauren e a idade média dos pacientes. Em relação à localização no estômago e ao estadiamento clínico, as neoplasias foram classificadas de acordo com a "Segunda Edição em Inglês da Classificação Japonesa do Carcinoma Gástrico".8 Em relação à localização, o estômago foi dividido em três segmentos: terço proximal (U), terço médio (M) e terço distal (L). Esses segmentos foram definidos pela subdivisão tanto da pequena quanto da grande curvatura em três partes com o mesmo comprimento. Foram incluídos no estudo somente os casos com lesões do terço médio do estômago ou do terço distal. No caso de lesão em ambas as porções foram classificadas como LM ou ML, dependendo da localização principal. Os casos de lesão do terço distal em que se observou acometimento do duodeno (D) foram classificados como LD. Em relação ao estadiamento os dados relacionados à profundidade da lesão $(\mathrm{T})$, presença de acomentimento de linfonodos $(\mathrm{N})$ e presença de metástases (M) também foram analisados isoladamente em relação à margem proximal.

Foram excluídos do estudo os casos de outras neoplasias gástricas que não o adenocarcinoma e os de acometimento do terço proximal do estômago. Foram excluídos da análise os dados incompletos no prontuário ou no laudo do exame de anatomia patológica.

A análise estatística foi realizada com o teste do qui-quadrado para proporções e o teste $t$ de student para médias. Foi considerado nível de significância de $5 \%$ $(P \leq 0,05)$.

\section{RESULTADOS}

Foram identificados 109 casos de neoplasia gástrica no período de estudo. Cinco casos foram excluídos: dois devido ao diagnóstico de GIST e linfoma gástrico e três em que foi realizada esofagogastrectomia. Foram incluídos 104 casos: 34 do sexo feminino e 70 do sexo masculino com idade média de $57 \pm 13$ anos. Não houve diferença de idade de acordo com o sexo dos pacientes (Tabela 1).
TABELA 1 - Dados demográficos dos adenocarcinomas gástricos

\begin{tabular}{llll}
\hline Sexo & n $(\%)$ & Idade & $\boldsymbol{P}$ \\
\hline Feminino & $34(32,7 \%)$ & $55,5 \pm 13$ anos & 0,22 \\
Masculino & $70(67,3 \%)$ & $59 \pm 13$ anos & \\
\hline Total & $\mathbf{1 0 4}$ & $\mathbf{5 7 , 9 \pm 1 3}$ anos & - \\
\hline
\end{tabular}

Os dados referentes à classificação de Lauren, classificação de Borrmann, diâmetro médio, localização, estadiamento clínico e presença de invasão angiolinfática estão representados na tabela 2. A maioria dos casos detectados foi de neoplasia do tipo intestinal da classificação de Lauren. Os portadores de neoplasia do tipo intestinal apresentaram idade média de $53 \pm 12$ anos, inferior à dos portadores de neoplasia do tipo difuso que foi de $61 \pm 13$ anos $(P=0,005)$.

Doze pacientes $(12,3 \%)$ apresentaram acometimento da margem proximal. A análise univariada entre os fatores analisados e o acometimento neoplásico da margem proximal demonstrou associação somente em relação à classificação de Borrmann (Tabela 2). Quanto mais elevado o estadiamento na classificação de Borrmann maior a porcentagem de acometimento da margem proximal. Todos os outros fatores analisados não demonstraram associação com o acometimento da margem proximal (Tabela 2).

TABELA 2 - Resultados dos fatores analisados e sua relação com o acometimento da margem proximal

\begin{tabular}{|c|c|c|c|c|}
\hline \multirow[b]{2}{*}{ Fator } & \multirow[b]{2}{*}{ n (\%) } & \multicolumn{2}{|c|}{ Margem } & \multirow[b]{2}{*}{$P$} \\
\hline & & $\begin{array}{l}\text { Livre } \\
\text { n (\%) }\end{array}$ & $\begin{array}{c}\text { Acometida } \\
\text { n (\%) }\end{array}$ & \\
\hline \multicolumn{5}{|l|}{ Classificação Lauren* } \\
\hline Intestinal & $59(58,4 \%)$ & $51(87,9 \%)$ & $7(12,1 \%)$ & 0,7 \\
\hline Difuso & $42(41,6 \%)$ & $36(85,7 \%)$ & $6(14,3 \%)$ & \\
\hline \multicolumn{5}{|l|}{ Classificação de Borrmann*, ** } \\
\hline I & $4(4,4 \%)$ & $3(75 \%)$ & $1(25 \%)$ & 0,003 \\
\hline II & $14(15,1 \%)$ & $14(100 \%)$ & $0(0 \%)$ & \\
\hline III & $63(67,7 \%)$ & $56(88,9 \%)$ & $7(11,1 \%)$ & \\
\hline IV & $10(10,6 \%)$ & $5(50 \%)$ & $5(50 \%)$ & \\
\hline $\mathrm{V}$ & $12(2,2 \%)$ & - & - & \\
\hline Diâmetro da lesão (média $\pm \mathrm{DP}) *$ & $5,2 \pm 2 \mathrm{~cm}$ & - & - & \\
\hline \multicolumn{5}{|l|}{ Diâmetro da lesão $(\leq 2 \mathrm{x}>2 \mathrm{~cm})^{*}$} \\
\hline$\leq 2 \mathrm{~cm}$ & $14(14 \%)$ & $11(78,5 \%)$ & $3(21,5 \%)$ & 0,17 \\
\hline$>2 \mathrm{~cm}$ & $86(86 \%)$ & $78(90,6 \%)$ & $8(9,4 \%)$ & \\
\hline \multicolumn{5}{|l|}{ Diâmetro da lesão $(\leq 4 \mathrm{x}>4 \mathrm{~cm}) *$} \\
\hline$\leq 4 \mathrm{~cm}$ & $43(43 \%)$ & $39(90,7 \%)$ & $4(9,3 \%)$ & 0,63 \\
\hline$>4 \mathrm{~cm}$ & $57(57 \%)$ & $50(87,7 \%)$ & $7(12,3 \%)$ & \\
\hline \multicolumn{5}{|l|}{ Localização da lesão* } \\
\hline M & $3(3,3 \%)$ & $72(92,3 \%)$ & $6(7,7 \%)$ & 0,29 \\
\hline ML & $9(9,8 \%)$ & $2(100 \%)$ & 0 & \\
\hline $\mathrm{L}$ & $78(84,8 \%)$ & $2(66,7 \%)$ & $1(33,3 \%)$ & \\
\hline LD & $2(2,1 \%)$ & $8(88,9 \%)$ & $1(11,1 \%)$ & \\
\hline \multicolumn{5}{|l|}{ clínico } \\
\hline Ia & $11(10,6 \%)$ & $11(100 \%)$ & 0 & 0,37 \\
\hline $\mathrm{Ib}$ & $14(13,5 \%)$ & $13(92,9 \%)$ & $1(7,1 \%)$ & \\
\hline II & $17(16,3 \%)$ & $14(82,4 \%)$ & $3(17,6 \%)$ & \\
\hline IIIa & $23(22,1 \%)$ & $22(95,7 \%)$ & $1(4,3 \%)$ & \\
\hline IIIb & $21(20,2 \%)$ & $16(80 \%)$ & $4(20 \%)$ & \\
\hline IV & $18917,3 \%)$ & $15(83,3 \%)$ & $3(16,7 \%)$ & \\
\hline \multicolumn{5}{|l|}{ Invasão angiolinfática* } \\
\hline Ausente & $33(34,7 \%)$ & $29(87,9 \%)$ & $4(12,1 \%)$ & 0,91 \\
\hline Presente & $62(65,3 \%)$ & $54(87,1 \%)$ & $8(12,9 \%)$ & \\
\hline
\end{tabular}

* Houve casos excluídos da análise por falta de dados; ** Os dois casos classificados como Borrmann $\mathrm{V}$ foram excluídos da análise sobre o acometimento da margem proximal; DP - desvio padrão 


\section{DISCUSSÃO}

A obtenção de margens livres de neoplasia é um princípio oncológico básico caso a intenção do procedimento seja curativa. Nos casos de adenocarcinoma gástrico do terço médio e distal tratados com gastrectomia subtotal a preocupação maior do cirurgião habitualmente está relacionada à margem cirúrgica distal. Nessa situação existe pequena extensão de duodeno disponível para a confecção de sutura adequada. Normalmente não se observa dificuldade em relação ao estabelecimento de margem proximal adequada. Os resultados do estadiamento pré-operatório associados à simples palpação e visualização do estômago durante o procedimento permitem avaliação adequada a respeito da extensão tumoral e determinação da margem proximal. Isso não é possível na gastrectomia videolaparoscópica. $\mathrm{O}$ cirurgião perde a capacidade de palpação o que torna mais difícil a determinação da margem cirúrgica proximal.

Diversos autores demonstraram acometimento neoplásico da margem proximal após as gastrectomias subtotais. Em um estudo a incidência foi de 8,2\% em 572 operações. 9 Outro estudo detectou acometimento da margem proximal em 1\% das 2740 gastrectomias subtotais avaliadas ${ }^{4}$. De Gara et al. ${ }^{5}$ demonstraram margem comprometida em 6\% dos 121 pacientes estádio 1 e 2 da classificação TNM; 22\% dos 132 pacientes com estádio 3 e $25 \%$ dos 60 pacientes com 4. Os autores incluíram nessa análise tanto a margem proximal quanto a distal em pacientes submetidos a gastrectomia subtotal e total.

Com o objetivo de evitar neoplasia residual alguns aspectos técnicos devem ser observados tanto nas gastrectomias abertas quanto nas laparoscópicas. Nos portadores de adenocarcinoma estádio III e especialmente estádio IV da classificação de Borrmann os limites do tumor não são muito bem definidos. Devido à possibilidade de neoplasia residual na margem proximal recomenda-se a realização de gastrectomia total nos portadores de carcinoma gástrico Borrmann IV IV $^{3,14}$. Nesse estudo, os estádios III e IV da classificação de Borrmann apresentaram acometimento da margem proximal em porcentagens significativas. Nos casos com margem positiva e grau IV de Borrmann, esse só foi constatado no exame patológico pós-operatório, mas não foi detectado na análise pré-operatória. Em relação aos portadores de carcinoma Borrmann I, a prevalência de acometimento da margem proximal foi de $25 \%$, superior ao observado nos portadores de Borrmann II e III $(0 \%$ e $11,1 \%$ respectivamente). Esse valor possivelmente está relacionado a erro estatístico devido ao baixo número de portadores de carcinoma Borrmann I nesse estudo.

A realização de gastrectomia total, denominada gastrectomia total de princípio, em todos os portadores de neoplasia gástrica, mesmo as do terço médio e distal e independente da classificação de Borrmann é outra conduta que visa evitar o acometimento da margem proximal. Trata-se de procedimento com morbidade superior a da gastrectomia subtotal, principalmente do ponto de vista nu- tricional e da qualidade de vida ${ }^{1,2,10}$. Essa conduta é bastante questionada porque estudos bem conduzidos demonstraram que a sobrevida dos pacientes submetidos à gastrectomia total ou subtotal é igual ${ }^{7}$. Bozzeti et al. randomizaram 618 pacientes portadores de adenocarcinoma gástrico da porção distal: 315 foram submetidos à gastrectomia subtotal e 303 a gastrectomia total ${ }^{1,2}$. Não houve diferença em relação à sobrevida em cinco anos, que foi de $65 \%$ e $62 \%$ respectivamente. Um paciente submetido à gastrectomia total e seis pacientes submetidos à gastrectomia subtotal apresentaram acometimento da margem proximal. Desses, em quatro casos a gastrectomia subtotal foi realizada com margem proximal de quatro centímetros e não de seis centímetros, o que foi condenado pelos autores. Indicam ainda a necessidade de gastrectomia total em virtualmente todos os pacientes portadores de tumores estádio T2 até T4 localizados no terço médio do estômago.

Alguns autores propõem a realização de biópsia de congelação nas margens proximais após a gastrectomia subtotal com a finalidade de detectar microscopicamente a presença de neoplasia residual ${ }^{9,11,14}$. Um estudo analisou a presença de neoplasia residual detectada em biópsia de congelação em 572 pacientes submetidos à gastrectomia ${ }^{9}$. Desses, 47 pacientes apresentaram margens positivas. A presença de neoplasia residual afetou o prognóstico na dependência da extensão da doença. Nos pacientes com mais de cinco linfonodos positivos na análise patológica a presença de margem proximal positiva não afetou o prognóstico. No entanto, nos pacientes com menos de cinco linfonodos acometidos a sobrevida foi negativamente afetada pela presença de margem proximal positiva. Matsusaka et al. ${ }^{11}$ realizaram biópsia de congelação da margem proximal em 34 pacientes submetidos à gastrectomia subtotal em que a análise pré-operatória não definiu adequadamente a margem de ressecção ${ }^{11}$. Em oito pacientes a biópsia foi positiva. O exame anátomo-patológico definitivo confirmou que houve acometimento da margem proximal em quatro. Nos outros quatro casos o exame anátomo-patológico definitivo foi negativo. Dos 26 casos com margem negativa na biópsia de congelação, todos foram negativos no exame anátomo-patológico definitivo. Uma recomendação interessante é a realização da biópsia de congelação somente nos casos de gastrectomia subtotal com intenção curativa. Nos casos com margem positiva durante a biópsia de congelação duas alternativas são possíveis: a) aumentar a margem de ressecção e realizar novamente a biópsia de congelação; b) realizar a gastrectomia total.

\section{CONCLUSÃO}

A classificação macroscópica de Borrmann, especialmente nos estádios III e IV, está relacionada à presença de acometimento da margem proximal nos casos de adenocarcinoma gástrico dos terços médio e distal submetidos a tratamento com gastrectomia subtotal. 
Freitas ACT, Dias JM, Okawa L, Coelho JCU. Proximal gastric margin analysis following subtotal gastrectomy for adenocarcinoma. ABCD Arq Bras Cir Dig 2008;21(3):106-9

ABSTRACT - Background - Subtotal gastrectomy is considered the gold standard treatment for gastric neoplasms localized in the distal and medial thirds of the stomach. Nevertheless, it has been shown that residual neoplasm into the proximal margin is associated to worse prognosis. Aim -To identify factors related to residual neoplasm into the proximal margin determined on pathology examination of patients subjected(Submitted) to subtotal gastrectomy for gastric adenocarcinoma. Methods - The charts of the patients subjected(Submitted) to subtotal gastrectomy due to gastric adenocarcinoma of the distal and medial thirds were reviewed from January 1998 to December 2007. It was recorded data referred to age, sex, Lauren and Borrmann staging, neoplasm diameter, localization inside the stomach, TNM staging and angiolymphatic invasion. These data were submitted to univariate analysis in relation to residual neoplasm into the proximal margin. Results - A hundread and four cases were included: 34 females and 70 males. The median age was $57 \pm 13$ years. Twelve patients $(12.3 \%)$ presented residual neoplasm into the proximal margin. The univariate analysis showed association between only the Borrmann staging and the residual neoplasm. Conclusion - Borrmann staging is associated to residual neoplasm into the proximal margin in patients subjected(Submitted) to subtotal gastrectomy for medial and distal thirds gastric adenocarcinoma.

HEADINGS - Stomach neoplasms. Adenocarcinoma. Gastrectomy. Neoplasm, residual.

\section{REFERÊNCIAS}

1. Bozzetti F, Marubini E, Bonfanti G, Miceli R, Piano C, Crose N. Total versus subtotal gastrectomy: surgical morbidity and mortality rates in a multicenter Italian randomized trial. The Italian Gastrointestinal Tumor Study Group. Ann Surg 1997;226:613-20.

2. Bozzetti F, Marubini E, Bonfanti G, Miceli R, Piano C, Gennari L. Subtotal versus total gastrectomy for gastric cancer: five-year survival rates in a multicenter randomized Italian trial. Italian Gastrointestinal Tumor Study Group. Ann Surg 1999;230:170-8.

3. Brennan MF. Current status of surgery for gastric cancer: a review. Gastric Cancer 2005, 8:64-70.

4. Cho BC, Jeung HC, Choi HJ, Rha SY, Hyung WJ, Cheong JH, Noh SH, Chung HC. Prognostic impact of resection margin involvement after extended (D2/ D3) gastrectomy for advanced gastric cancer: a 15-year experience at a single institute. J Surg Oncol 2007; 1:461-8.

5. De Gara CJ,Hanson J, Hamilton S. A population-based study of tumor-node relationship, resection margins, and surgeon volume on gastric cancer survival. Am J Surg 2003, 186:23-7.

6. Ferlay J, Bray F, Pisani P, Parkin DM. GLOBOCAN 2002: Cancer Incidence, Mortality and Prevalence Worldwide. Lyon, IARC Press, 2004.

7. Gouzi JL, Huguier M, Fagniez PL, Launois B, Flamant Y, Lacaine F. Total versus subtotal gastrectomy for adenocarcinoma of the gastric antrum. A French prospective controlled study. Ann Surg 1989;209:162-6.

8. Japanese Classification of Gastric Carcinoma - 2nd English Edition - Japanese Gastric Cancer Association. Gastric Cancer 1998; 1:10-24.
9. Kim SH, Karpeh MS, Klimstra DS, Leung D, Brennan MF. Effect of microscopic resection line disease on gastric cancer survival. J Gastrointest Surg 1999;3:24-33.

10. Machi J, Takeda J, Kakegawa T. Tumores do estômago e do duodeno. In: Coelho JCU. Aparelho Digestivo. Clínica e Cirurgia. 3.ed. São Paulo, Editora Atheneu, 2005.

11. Matsusaka S, Nagareda T, Yamasaki H, Kitayama Y, Okada T, Maeda S. Immunohistochemical Evaluation for Intraoperative Rapid Pathological Assessment of the Gastric Margin. World J Surg 2003;27:715-8.

12. Robertson CS, Chung SC, Woods SD, Griffin SM, Raimes SA, Lau JT. A prospective randomized trial comparing R1 subtotal gastrectomy with R3 total gastrectomy for antral cancer. Ann Surg 1994; 220:176-82.

13. Rohde H, Gebbensleben B, Bauer P, Stutzer H, Zieschang J. Has there been any improvement in the staging of gastric cancer? Findings from the German Gastric Cancer TNM Study Group. Cancer 1989;64:2465-81.

14. Siewert JR, Hofler H, Fink U, Mueller J, SEndler A, Molls M, Becker K, Nekarda H, Bottcher K, Roder JD, Feldman HJ, Stein HJ. Curr Probl Surg 2006; 43:566-670.

Fonte de financiamento: não há Conflito de interesse: não há Recebido para publicação: 10/02/2008 Aceito para publicação: 30/04/2008 